\title{
Epitaxial contact Andreev reflection spectroscopy of $\mathrm{NbN} / \mathrm{Co}_{2} \mathrm{FeSi}$ layered devices
}

Iduru Shigeta, Takahide Kubota, Yuya Sakuraba, Cor G. Molenaar, Joost N. Beukers, Shojiro Kimura, Alexander A. Golubov, Alexander Brinkman, Satoshi Awaji, Koki Takanashi, and Masahiko Hiroi

Citation: Appl. Phys. Lett. 112, 072402 (2018); doi: 10.1063/1.5007287

View online: https://doi.org/10.1063/1.5007287

View Table of Contents: http://aip.scitation.org/toc/apl/112/7

Published by the American Institute of Physics

\section{Articles you may be interested in}

Magnetic tunnel junctions with an equiatomic quaternary CoFeMnSi Heusler alloy electrode

Applied Physics Letters 112, 052403 (2018); 10.1063/1.5002763

Large moments in bcc $\mathrm{Fe}_{\mathrm{x}} \mathrm{Co}_{\mathrm{y}} \mathrm{Mn}_{\mathrm{z}}$ ternary alloy thin films

Applied Physics Letters 112, 072403 (2018); 10.1063/1.5006347

Perpendicular magnetic tunnel junctions with Mn-modified ultrathin MnGa layer

Applied Physics Letters 112, 062402 (2018); 10.1063/1.5002616

Mobility spectrum analytical approach for the type-II Weyl semimetal $\mathrm{T}_{d}-\mathrm{MoTe}_{2}$

Applied Physics Letters 112, 072401 (2018); 10.1063/1.5008850

Precision measurement of the quantized anomalous Hall resistance at zero magnetic field

Applied Physics Letters 112, 072102 (2018); 10.1063/1.5009718

Temperature dependence of interlayer coupling in perpendicular magnetic tunnel junctions with $\mathrm{GdO}$ Xarriers Applied Physics Letters 112, 072404 (2018); 10.1063/1.5002586

\section{PHYSICS TODAY}

MANAGER'S GUIDE

WHITEPAPERS
Accelerate R\&D with

Multiphysics Simulation
READ NOW

PRESENTED BY

И $\subset$ OMSOL 


\title{
Epitaxial contact Andreev reflection spectroscopy of $\mathrm{NbN} / \mathrm{Co}_{2} \mathrm{FeSi}$ layered devices
}

\author{
Iduru Shigeta, ${ }^{1, a)}$ Takahide Kubota, ${ }^{2,3}$ Yuya Sakuraba, ${ }^{4}$ Cor G. Molenaar, ${ }^{5}$ \\ Joost N. Beukers, ${ }^{5}$ Shojiro Kimura, ${ }^{6}$ Alexander A. Golubov, ${ }^{5,7}$ Alexander Brinkman, ${ }^{5}$ \\ Satoshi Awaji, ${ }^{6}$ Koki Takanashi, ${ }^{2,3}$ and Masahiko Hiroi ${ }^{1}$ \\ ${ }^{1}$ Department of Physics and Astronomy, Graduate School of Science and Engineering, Kagoshima University, \\ Korimoto 1-21-35, Kagoshima 890-0065, Japan \\ ${ }^{2}$ Institute for Materials Research, Tohoku University, Katahira 2-1-1, Aoba-ku, Sendai 980-8577, Japan \\ ${ }^{3}$ Center for Spintronics Research Network (CSRN), Tohoku University, Katahira 2-1-1, Aoba-ku, \\ Sendai 980-8577, Japan \\ ${ }^{4}$ National Institute for Materials Science (NIMS), Sengen 1-2-1, Tsukuba 305-0047, Japan \\ ${ }^{5}$ Faculty of Science and Technology and MESA+ Institute for Nanotechnology, University of Twente, \\ 7500 AE Enschede, The Netherlands \\ ${ }^{6}$ High Field Laboratory for Superconducting Materials, Institute for Materials Research, Tohoku University, \\ Katahira 2-1-1, Aoba-ku, Sendai 980-8577, Japan \\ ${ }^{7}$ Moscow Institute of Physics and Technology, Dolgoprudny, Moscow Region 141700, Russia
}

(Received 2 October 2017; accepted 29 January 2018; published online 12 February 2018)

\begin{abstract}
We investigated the spin polarization $P$ of Co-based Heusler alloy $\mathrm{Co}_{2} \mathrm{FeSi}$ by epitaxial contact Andreev reflection (ECAR) spectroscopy using epitaxially grown superconductor $\mathrm{NbN}$ and Heusler alloy $\mathrm{Co}_{2} \mathrm{FeSi}$ layered devices. Ferromagnetic $\mathrm{Co}_{2} \mathrm{FeSi}$ possesses the highest Curie temperature $\left(T_{\mathrm{C}}\right.$ $\approx 1100 \mathrm{~K})$ and the largest spontaneous magnetic moment $\left(p_{\mathrm{s}} \approx 6 \mu_{\mathrm{B}}\right)$ in the class of Heusler alloys. The ECAR measurements revealed that the $P$ value of $\mathrm{Co}_{2} \mathrm{FeSi}$ was $54 \pm 2 \%$ with a finite barrier parameter $Z$, indicating that an intrinsic $P$ value in ECAR spectroscopy would exceed reported values in point-contact Andreev reflection spectroscopy. We therefore established not only the epitaxial integration of ferromagnetic $\mathrm{Co}_{2} \mathrm{FeSi}$ with superconductor $\mathrm{NbN}$ on an $\mathrm{MgO}$ substrate but also the fabrication and evaluation techniques of their ECAR devices. This highly versatile superconducting spintronic system enables fundamental superconducting spintronic studies, and it is also a candidate for practical superconducting spintronic devices. Published by AIP Publishing. https://doi.org/10.1063/1.5007287
\end{abstract}

The study of superconducting and ferromagnetic bilayers, trilayers, and multilayers has great importance due to their potential applications ranging from fundamental superconducting spintronic studies ${ }^{1,2}$ to practical superconducting spintronic devices. ${ }^{3-5}$ The macroscopic quantum states in superconducting and ferromagnetic layers influence each other via the penetration of superconducting order (proximity effect) ${ }^{1}$ and ferromagnetic order (inverse proximity effect $)^{2}$ through their common interface. Ferromagnetic components consisting of $3 d$ transition metals like $\mathrm{Fe}$, Co, $\mathrm{Ni}$, and their alloys were commonly used for the study stated above. In an attempt to realize superconducting spintronic devices, there is therefore a clear need for a spin injector material with high spin polarization and a good lattice matching to be epitaxially grown onto the working superconductor. One such potential candidate is the highly spin polarized Co-based Heusler alloys, whose epitaxial integration with superconductor $\mathrm{NbN}$ should yield high injectionefficiency spin-selective ohmic contacts, thereby enabling the development of practical superconducting spintronic devices, such as the spin Josephson junction ${ }^{4,6,7}$ and superconducting spin transistor ${ }^{8,9}$ devices, with both low power consumptions and high speeds.

A number of studies using Co-based Heusler alloys have recently achieved large tunneling magnetoresistance (TMR)

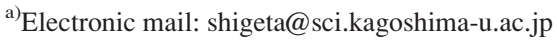

and giant magnetoresistance (GMR) effects originating from the half-metallic nature of Co-based Heusler alloys. Giant TMR ratios over $2600 \%$ at low temperature have been reported in magnetic tunnel junctions (MTJs) with epitaxial $\mathrm{Co}_{2} \mathrm{Mn}_{1.24} \mathrm{Fe}_{0.16} \mathrm{Si}_{0.84}$ electrodes with the crystalline $\mathrm{MgO}$ barriers. ${ }^{10}$ Large MR ratios over $30 \%$ at room temperature (RT) have also been observed in current-perpendicularto-plane giant magnetoresistive (CPP-GMR) devices using epitaxial $\mathrm{Co}_{2} \mathrm{Fe}_{0.4} \mathrm{Mn}_{0.6} \mathrm{Si}$ ferromagnetic electrodes, ${ }^{11,12}$ which are one order of magnitude higher than those reported in the CPP-GMR devices with conventional ferromagnetic layers such as $\mathrm{CoFe}$ or NiFe electrodes. In contrast, only few reports exist for epitaxial $\mathrm{NbN} / \mathrm{He}$ usler alloy layered films and their devices so far; for example, giant coercivity enhancement in $\mathrm{NbN} / \mathrm{Co}_{2} \mathrm{FeSi}$ bilayers, ${ }^{13}$ efficient injection of pure spin currents in $\mathrm{NbN} / \mathrm{Co}_{2} \mathrm{MnSi}$ devices ${ }_{15}^{14}$ superconductivity in $\mathrm{NbN} / \mathrm{Co}_{2} \mathrm{Fe}_{0.4} \mathrm{Mn}_{0.6} \mathrm{Si} / \mathrm{Au}$ trilayers, ${ }^{15}$ and investigation of odd frequency pairs generated in highly spin polarized ferromagnetic Josephson devices. ${ }^{16}$

Point-contact Andreev reflection (PCAR) spectroscopy is an alternative powerful technique to evaluate the spin polarization of half-metallic ferromagnets as it is simple to fabricate junctions and to apply model calculations. ${ }^{17-19}$ The large values of the spin polarization in half-metallic Heusler alloys were reported to be $74 \pm 2 \%$ for $\mathrm{Co}_{2} \mathrm{MnGe}_{0.75} \mathrm{Ga}_{0.25}{ }^{20}$ and $72 \pm 2 \%$ for $\mathrm{Co}_{2} \mathrm{MnGa}_{0.5} \mathrm{Sn}_{0.5}{ }^{21}$ in PCAR spectroscopy. In principle, the contact interface in PCAR spectroscopy is 
not necessarily clean at the atomic scale because (i) the sample surface is contaminated when exposed to the air before making a point contact, while during PCAR spectroscopy, the oxide layers presented on both the superconducting tip and the ferromagnet create microcracks which act as ballistic tunnel paths for the electrons; (ii) a point contact with a superconducting tip may give rise to crystal structure destruction at the contact interface. Although such crystal disorders at the contact interface are possible to degrade intrinsic high spin polarization of Heusler alloys,${ }^{22}$ there are no reports for spin polarization measurements of Heusler alloys by epitaxial contact Andreev reflection (ECAR) spectroscopy using epitaxially grown devices including a clean $\mathrm{NbN} /$ Heusler alloy interface.

In this letter, we investigated not only the structural, magnetic, and transport properties of epitaxial $\mathrm{NbN} / \mathrm{Co}_{2} \mathrm{FeSi} / \mathrm{Au}$ trilayer films but also the spin polarization of their ECAR devices. $\mathrm{NbN}$ is a type-II superconductor of the high critical temperature $\left(T_{\mathrm{c}} \approx 16 \mathrm{~K}\right)$, the short coherence length $[\xi(0) \approx 5 \mathrm{~nm}]$, and the long penetration depth $[\lambda(0) \approx 200 \mathrm{~nm}] .{ }^{23,24}$ Co-based Heusler alloy $\mathrm{Co}_{2} \mathrm{FeSi}$ possesses the highest Curie temperature $\left(T_{\mathrm{C}} \approx 1100 \mathrm{~K}\right)$ and the largest spontaneous magnetic moment $\left(p_{\mathrm{s}} \approx 6 \mu_{\mathrm{B}}\right)$ in the class of Heusler alloys. ${ }^{25}$ We have fabricated the $\mathrm{NbN} / \mathrm{Co}_{2} \mathrm{FeSi}$ layered devices including ultra-clean interfaces and evaluated the spin polarization of $\mathrm{Co}_{2} \mathrm{FeSi}$ by ECAR spectroscopy.

Epitaxially grown layered samples including the $\mathrm{NbN} /$ $\mathrm{Co}_{2} \mathrm{FeSi}$ interface were prepared using an ultra-high vacuum (UHV)-compatible magnetron sputtering system with a base pressure below $1 \times 10^{-7} \mathrm{~Pa}$. The surface of an $\mathrm{MgO}$ substrate was thermally cleaned in the UHV chamber before depositing $\mathrm{NbN}$ and $\mathrm{Co}_{2} \mathrm{FeSi}$ layers. First, a superconducting $\mathrm{NbN}$ layer was directly grown at $\mathrm{RT}$ on an $\mathrm{MgO}$ single-crystal substrate using a niobium target by reactive sputtering in nitrogen and argon mixture gas. A Heusler alloy $\mathrm{Co}_{2} \mathrm{FeSi}$ layer was also deposited at RT followed by in situ annealing at $450^{\circ} \mathrm{C}$ to promote chemical ordering. The thicknesses of the $\mathrm{NbN}$ and $\mathrm{Co}_{2} \mathrm{FeSi}$ layers were controlled to be $100 \mathrm{~nm}$ and $5 \mathrm{~nm}$, respectively. After the film was cooled to RT, the film was finally capped by a 3 -nm-thick Au protective layer. All the procedures were continuously carried out inside the UHV chambers without breaking the vacuum to maintain the clean interfaces.

Fabricated trilayer films were patterned into a pillar shape for a CPP-type four-terminal device structure using electron beam lithography and $\mathrm{Ar}$ ion milling. ${ }^{12}$ The designed diameter of circular pillars changed from $60 \mathrm{~nm}$ to $4 \mu \mathrm{m}$ on one substrate. The differential conductance $G(V)$ of the $\mathrm{NbN} / \mathrm{Co}_{2} \mathrm{FeSi}$ layered ECAR devices was measured by standard ac lock-in detection at a frequency of $1 \mathrm{kHz}$. The normalized conductance curve $G(V) / G_{\mathrm{N}}$ was fitted to the modified Blonder-Tinkham-Klapwijk (BTK) model $^{18}$ in order to evaluate the spin polarization $P$ of the conductive $s p$-electrons in the Heusler alloy $\mathrm{Co}_{2} \mathrm{FeSi}$. A multiple parameter fitting was carried out using the spin polarization $P$, the superconducting energy gap $\Delta$, the dimensionless barrier parameter $Z$, and the broadening parameter $\omega$ as the variable parameters. ${ }^{26}$

The crystal structure of $\mathrm{NbN} / \mathrm{Co}_{2} \mathrm{FeSi} / \mathrm{Au}$ trilayer films was confirmed at RT by X-ray diffraction (XRD) using monochromatic $\mathrm{Cu}-K \alpha$ radiation and reflection high-energy electron diffraction (RHEED). Figure 1(a) shows the $2 \theta$ profile for a $\mathrm{NbN} / \mathrm{Co}_{2} \mathrm{FeSi} / \mathrm{Au}$ trilayer film. The result of the $2 \theta$ profile exhibits that the $\mathrm{NbN}$ layer is single phase within the resolution limits of the XRD. Figure 1(b) shows the rocking curve of the $\mathrm{NbN}(002)$ peak in Fig. 1(a). A full-width at half-maximum (FWHM) of $1.55^{\circ}$ in the rocking curve suggests that the strain of the epitaxially grown $\mathrm{NbN}(001)$ layer on the $\mathrm{MgO}$ substrate is relatively small when compared to $\mathrm{NbN}$ films of different nitrogen concentrations with lower $T_{\mathrm{c} .}{ }^{27}$ Here, we note that there are no Bragg reflections for the $\mathrm{Co}_{2} \mathrm{FeSi}$ and $\mathrm{Au}$ layers in Fig. 1(a) because of the thin layer thickness of the order of a few nanometers which might be under the detection limit of the XRD facility. The epitaxial growth and the chemical ordering of the $\mathrm{Co}_{2} \mathrm{FeSi}$ layer were therefore evaluated by using RHEED images. Figures 2(a) and 2(b) show the RHEED images of a $\mathrm{Co}_{2} \mathrm{FeSi}$ layer surface along $\mathrm{Co}_{2} \mathrm{FeSi}[100]$ and $\mathrm{Co}_{2} \mathrm{FeSi}[110]$ directions. The RHEED images denote that the superlattice diffraction lines [arrows in Fig. 2(b)] from the $L 2_{1}$-ordered phase of $\mathrm{Co}_{2} \mathrm{FeSi}$ were confirmed when an electron-beam was incident to the
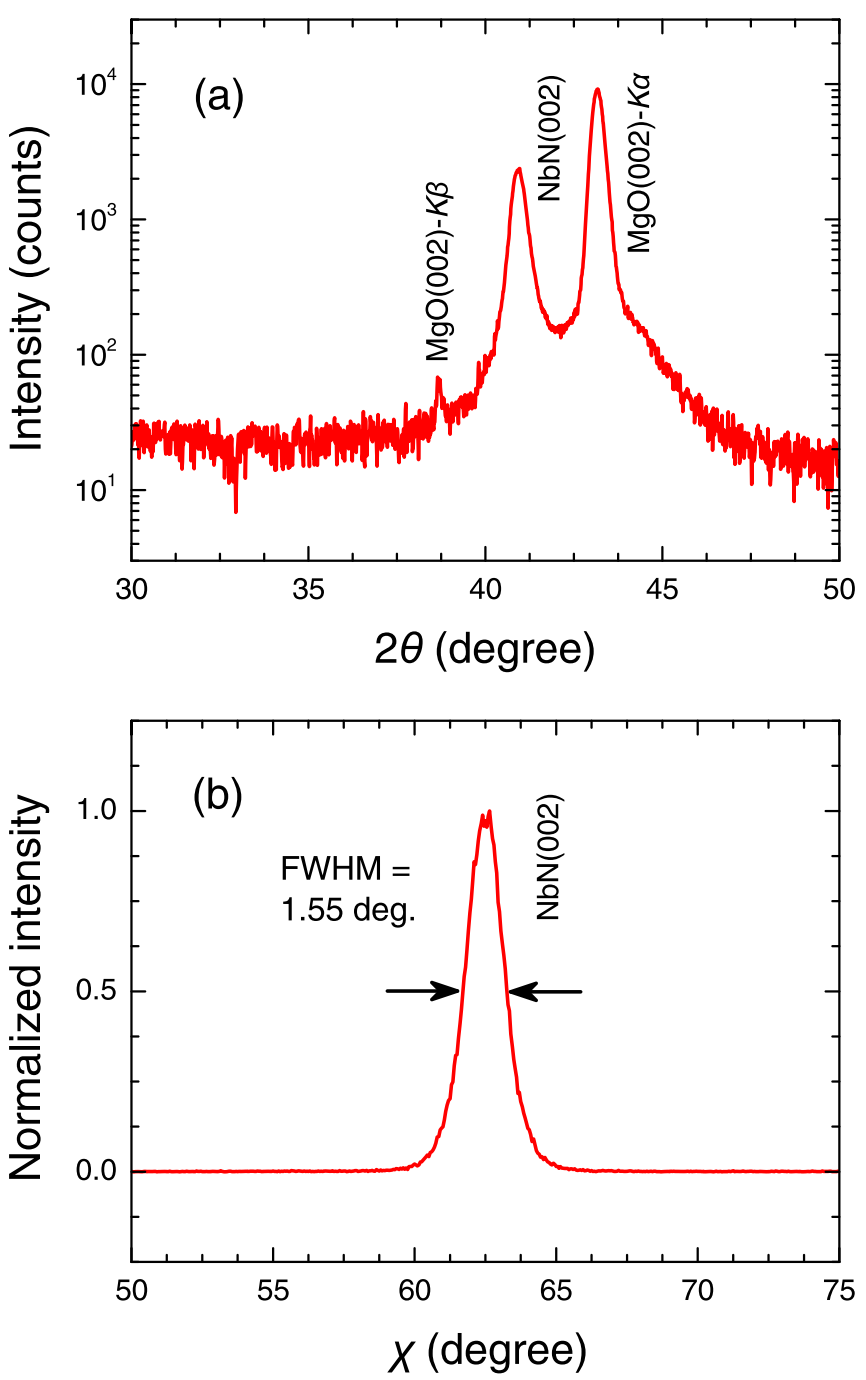

FIG. 1. XRD patterns of the (a) $2 \theta$ profile and (b) rocking curve of the $\mathrm{NbN}(002)$ reflection in a $\mathrm{NbN} / \mathrm{Co}_{2} \mathrm{FeSi} / \mathrm{Au}$ trilayer film on an $\mathrm{MgO}$ substrate. The plots demonstrate the epitaxial growth of the $\mathrm{NbN}$ layer with a high crystalline quality and the absence of second phases within the resolution limits of the XRD. 

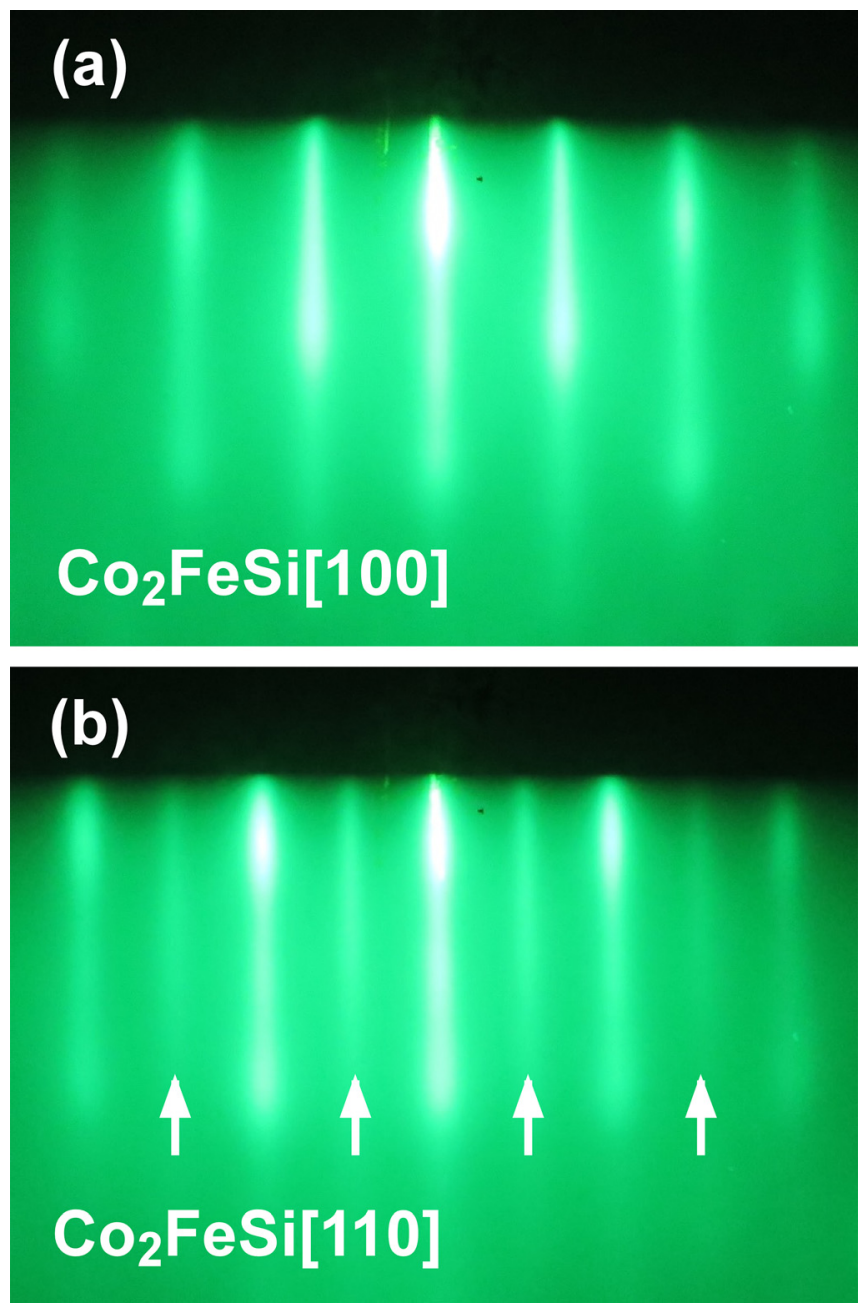

FIG. 2. RHEED images obtained from the surface of a $\mathrm{Co}_{2} \mathrm{FeSi}$ layer with the electron beam parallel to (a) [100] and (b) [110] directions of the $\mathrm{Co}_{2} \mathrm{FeSi}$ layer. The superlattice diffractions originating from the $L 2_{1}$-phase are pointed with the arrows.

$\mathrm{Co}_{2} \mathrm{FeSi}[110]$ direction of the surface. The XRD and RHEED measurements demonstrate the epitaxial growth of both $\mathrm{NbN}$ and $\mathrm{Co}_{2} \mathrm{FeSi}$ layers. From the XRD patterns and RHEED images, the epitaxial relationships of the layered sample were confirmed as follows, from the substrate to top: $\mathrm{MgO}(001)[100]|\mathrm{NbN}(001)[100]| \mathrm{Co}_{2} \mathrm{FeSi}(001)[110]$.

The magnetization $M(H)$ curves of the $\mathrm{NbN} / \mathrm{Co}_{2} \mathrm{FeSi} / \mathrm{Au}$ trilayer film were investigated at RT by using a vibrating sample magnetometer (VSM). The magnetic properties of the trilayer film arise from the $\mathrm{Co}_{2} \mathrm{FeSi}$ layer, which is ferromagnetic at $\mathrm{RT}$ while the $\mathrm{NbN}$ and Au layers are paramagnetic. Figure 3 shows the $M(H)$ curves measured for an unpatterned trilayer film with a 5-nm-thick $\mathrm{Co}_{2} \mathrm{FeSi}$ layer. The magnetic field was applied along the $\mathrm{Co}_{2} \mathrm{FeSi}[100]$ (blue line) and $\mathrm{Co}_{2} \mathrm{FeSi}[110]$ (red line) directions, respectively. The magnetization was almost saturated at $100 \mathrm{Oe}$, and the saturation magnetization $M_{\mathrm{s}}$ was obtained to be $1200 \mathrm{emu} / \mathrm{cm}^{3}$. This value rivals that of the $\mathrm{Co}_{2} \mathrm{FeSi}$ bulk, ${ }^{28}$ and it is also close to the theoretical prediction of the spontaneous magnetic moment $p_{\mathrm{s}}=6 \mu_{\mathrm{B}} / \mathrm{f}$.u. for the Slater-Pauling rule. ${ }^{29}$ Small but finite magnetocrystalline anisotropy was found in the $\mathrm{Co}_{2} \mathrm{FeSi}$ film along the easy axis of the $\mathrm{Co}_{2} \mathrm{FeSi}[100]$ direction, and the coercivity $H_{\mathrm{c}}$ was $27 \mathrm{Oe}$.

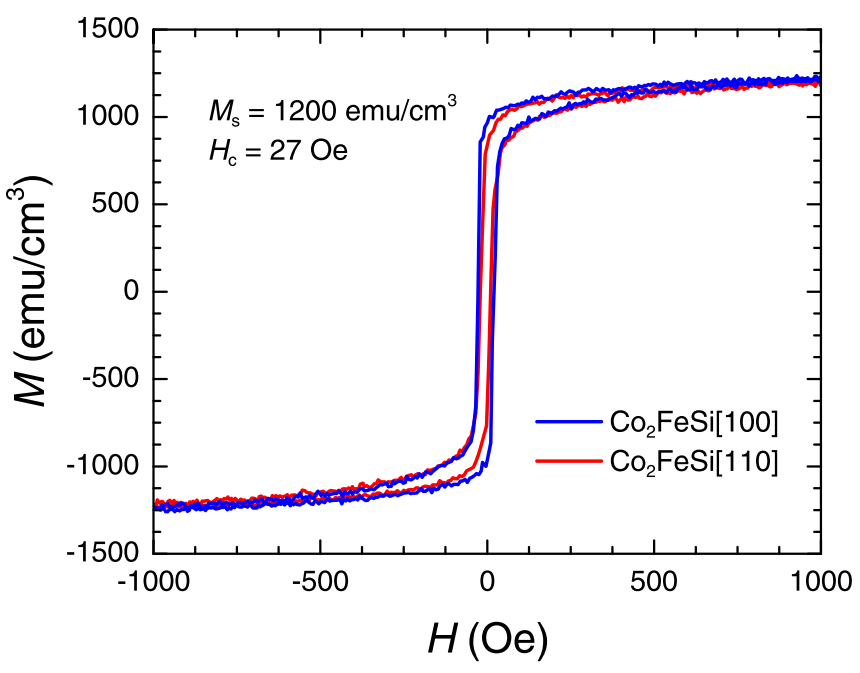

FIG. 3. Magnetization $M(H)$ curves measured for the $\mathrm{NbN} / \mathrm{Co}_{2} \mathrm{FeSi} / \mathrm{Au}$ trilayer film with a 5 -nm-thick $\mathrm{Co}_{2} \mathrm{FeSi}$ layer. The blue and red lines correspond to the $M(H)$ curves along the $\mathrm{Co}_{2} \mathrm{FeSi}[100]$ and the $\mathrm{Co}_{2} \mathrm{FeSi}[110]$ directions, respectively. The in-plane magnetic easy-axis is the $\mathrm{Co}_{2} \mathrm{FeSi}[100]$ direction.

Before patterning into the device structure, the resistivity $\rho(T)$ of the $\mathrm{NbN} / \mathrm{Co}_{2} \mathrm{FeSi} / \mathrm{Au}$ trilayer film was measured by the dc four-terminal method under magnetic fields up to $17 \mathrm{~T}$ in the temperature range between $4.2 \mathrm{~K}$ and $300 \mathrm{~K}$. Figure 4 shows temperature-dependent $\rho(T)$ for magnetic fields applied parallel to the surface of a $\mathrm{NbN} / \mathrm{Co}_{2} \mathrm{FeSi} / \mathrm{Au}$ trilayer film, varying in $1 \mathrm{~T}$ steps from 0 to $17 \mathrm{~T}$. As shown in Fig. 4, the superconducting transition temperature $T_{\mathrm{c}}$ decreased monotonically with the increase in the magnetic fields, while the superconducting transition width $\Delta T_{\mathrm{c}}$ was almost unchanged. In Fig. 4, we obtained that the normal resistivity $\rho_{20 \mathrm{~K}}$ at $20 \mathrm{~K}$ was $69.2 \mu \Omega \cdot \mathrm{cm}$ and the residual resistivity ratio (RRR) was $1.12 . T_{\mathrm{c}}$ was defined as the temperature at which the resistivity dropped to half its normal state value and it was determined to be $16.3 \mathrm{~K}$ in the absence

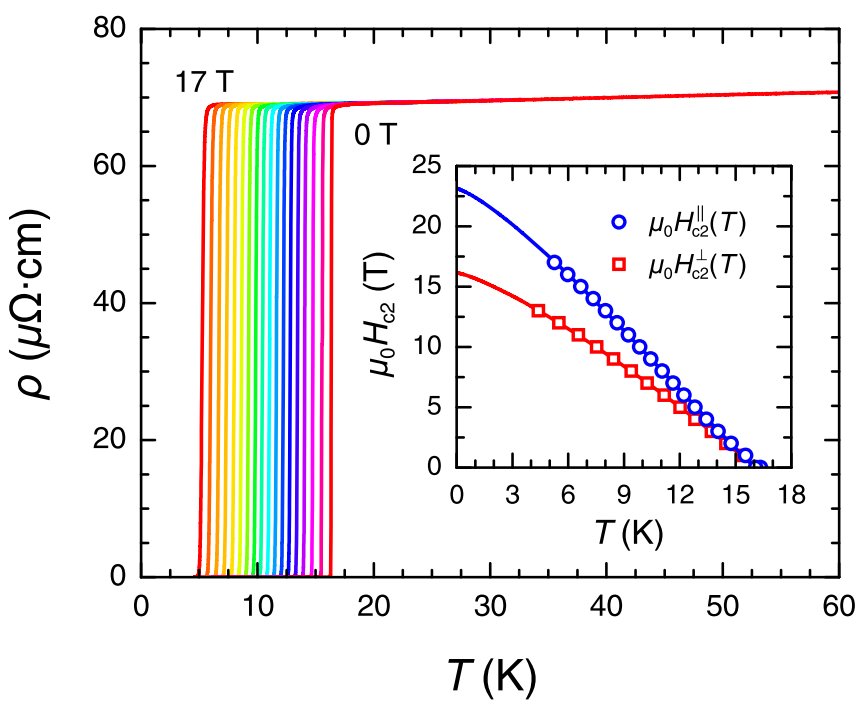

FIG. 4. Temperature dependence of resistivity $\rho(T)$ of a $\mathrm{NbN} / \mathrm{Co}_{2} \mathrm{FeSi} / \mathrm{Au}$ trilayer film for the parallel magnetic fields, varying in $1 \mathrm{~T}$ steps from 0 to $17 \mathrm{~T}$. The inset of Fig. 4 is the temperature dependence of the upper critical fields $\mu_{0} H_{\mathrm{c} 2}^{\|}(T)$ and $\mu_{0} H_{\mathrm{c} 2}^{\perp}(T)$ in two magnetic fields parallel and perpendicular to the surface of the trilayer film. The blue and red lines are theoretical curves obtained from the BCS-type function. 
of magnetic fields, which coincided with that of our 100-nmthick $\mathrm{NbN}$ monolayer films. In addition, $T_{\mathrm{c}}$ is comparable to that of the maximum value for $\mathrm{NbN}$ films deposited at $\mathrm{RT} .^{24}$

The upper critical field $\mu_{0} H_{\mathrm{c} 2}(T)$ was also evaluated from the $\rho(T)$ measurements in Fig. 4. The inset of Fig. 4 presents the temperature dependence of the upper critical fields $\mu_{0} H_{\mathrm{c} 2}^{\|}(T)$ and $\mu_{0} H_{\mathrm{c} 2}^{\perp}(T)$ of a $\mathrm{NbN} / \mathrm{Co}_{2} \mathrm{FeSi} / \mathrm{Au}$ trilayer film in two magnetic field directions applied parallel and perpendicular to the surface of the trilayer film. For the outof-plane magnetic fields, $T_{\mathrm{c}}$ is rapidly suppressed with a broadening of the superconducting transition, which is in contrast to those observed in the in-plane magnetic fields. Such an anisotropy is ascribed to 2-dimensional (2D) geometry of the film, leading to contribution of the vortex motion in the out-of-plane magnetic field. The upper critical field $\mu_{0} H_{\mathrm{c} 2}(0)$ at $0 \mathrm{~K}$ was determined from the Bardeen-Cooper-Schrieffer (BCS)-type function $\mu_{0} H_{\mathrm{c} 2}(T)=\mu_{0} H_{\mathrm{c} 2}(0)\left[1-\left(T / T_{\mathrm{c}}\right)^{\alpha}\right]^{\beta}$, using the trilayer film's $T_{\mathrm{c}}$ with $\mu_{0} H_{\mathrm{c} 2}(0)$ and $\alpha$ and $\beta$ as fitting parameters. ${ }^{30} \mathrm{We}$ found $\mu_{0} H_{\mathrm{c} 2}^{\|}(0)=23.2 \mathrm{~T}$ with $\alpha=1.26$ and $\beta=1.09$ in the parallel magnetic field (blue line) and $\mu_{0} H_{\mathrm{c} 2}^{\perp}(0)=16.2 \mathrm{~T}$ with $\alpha=1.26$ and $\beta=1.00$ in the perpendicular magnetic field (red line). Considering that the thickness $d_{\mathrm{NbN}}$ of the NbN layer is $100 \mathrm{~nm}$ and its coherence length $\xi_{\mathrm{NbN}}(0)$ at $0 \mathrm{~K}$ is about $5 \mathrm{~nm}$, the superconductivity of the trilayer film should be in the three-dimensional (3D) regime, due to satisfying $d_{\mathrm{NbN}} \gg \xi_{\mathrm{NbN}}(0)$. Indeed, $\mu_{0} H_{\mathrm{c} 2}(T) \propto 1-T / T_{\mathrm{c}}$ was substantially fulfilled in both magnetic field directions, corresponding to $\alpha=1$ and $\beta=1$ in the $3 \mathrm{D}$ regime. ${ }^{31}$

The spin polarization of ferromagnetic Heusler alloy $\mathrm{Co}_{2} \mathrm{FeSi}$ was evaluated using $\mathrm{NbN} / \mathrm{Co}_{2} \mathrm{FeSi}$ layered ECAR devices, which were patterned from the trilayer films excellent in magnetic and superconducting properties. The differential conductance $G(V)$ of the ECAR devices, whose interface was well-defined at the atomic scale, was measured by ECAR spectroscopy. In this study, at least 20 different devices ranging in diameter from $60 \mathrm{~nm}$ to $1 \mu \mathrm{m}$ were measured, whereas $G(V)$ was obtained for less than 100-nmdiameter devices. It was difficult for large size devices to apply bias voltage beyond the superconducting energy gap $\Delta$ because their device resistance decreased with the increase in the pillar diameter.

With regard to the ECAR devices analyzed by the modified BTK model, the typical device resistance is about $10 \Omega$. Figure 5 shows the analytical result of the normalized $G(V) /$ $G_{\mathrm{N}}$ for a $\mathrm{NbN} / \mathrm{Co}_{2} \mathrm{FeSi}$ layered ECAR device at $4.2 \mathrm{~K}$, indicating that the experimentally obtained $G(V) / G_{\mathrm{N}}$ was in good agreement with that of the modified BTK model except for the region of the dip structure. As shown in Fig. 5, the peak and dip structures were observed at $2.33 \mathrm{mV}$ and $4.13 \mathrm{mV}$, respectively. The temperature evolution of $G(V)$ was also measured to investigate the origin of the peak and dip structures. Figure 6 presents the intensity plot of $G(V) / G_{\mathrm{N}}$ as a function of temperature and applied bias voltage for the same device in Fig. 5. We found that the peak and dip structures disappeared above $T_{\mathrm{c}}$, indicating that these structures originate from the superconductivity. Hence, the peak corresponds to the superconducting energy gap $\Delta$, and the dip structure might be attributed to the superconducting critical current effect. ${ }^{32}$

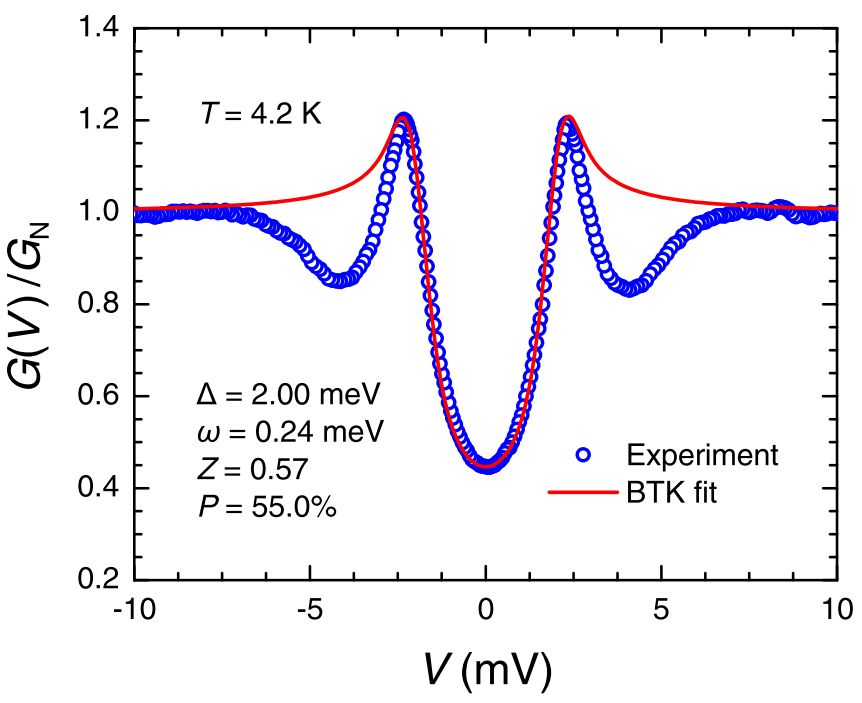

FIG. 5. Fitting result of the modified BTK analysis for the normalized differential conductance $G(V) / G_{\mathrm{N}}$ of a $\mathrm{NbN} / \mathrm{Co}_{2} \mathrm{FeSi}$ layered ECAR device at $4.2 \mathrm{~K}$. The experimentally obtained $G(V) / G_{\mathrm{N}}$ was in good agreement with the fitting curve for the modified BTK theory except for the region of the dip structure.

From the modified BTK analysis in Fig. 5, the spin polarization was determined to be $P=55.0 \%$ with $\Delta=2.00 \mathrm{meV}, \omega=0.24 \mathrm{meV}$, and $Z=0.57$, as fitting parameters. In general, the intrinsic $P$ value of ferromagnets can be deduced by extrapolating $P$ down to $Z=0$ in the $P(Z)$ plot. $^{20,33}$ However, since Figs. S1(a)-S1(c) (supplementary material) exhibit that the conductance spectral shapes were almost reproducible in the different devices on the same substrate and the $P$ values were practically independent of $Z$ ranging between 0.5 and 0.6 , the $P$ values for individual ECAR devices were averaged out. We found the average value of the spin polarization $\langle P\rangle=54 \pm 2 \%$. Although it was reported that the intrinsic $P$ values of $\mathrm{Co}_{2} \mathrm{FeSi}$ ranged from $49 \%$ to $55 \%$ in PCAR spectroscopy, ${ }^{34-37}$ the intrinsic $P$ value of $\mathrm{Co}_{2} \mathrm{FeSi}$ in ECAR spectroscopy would exceed the

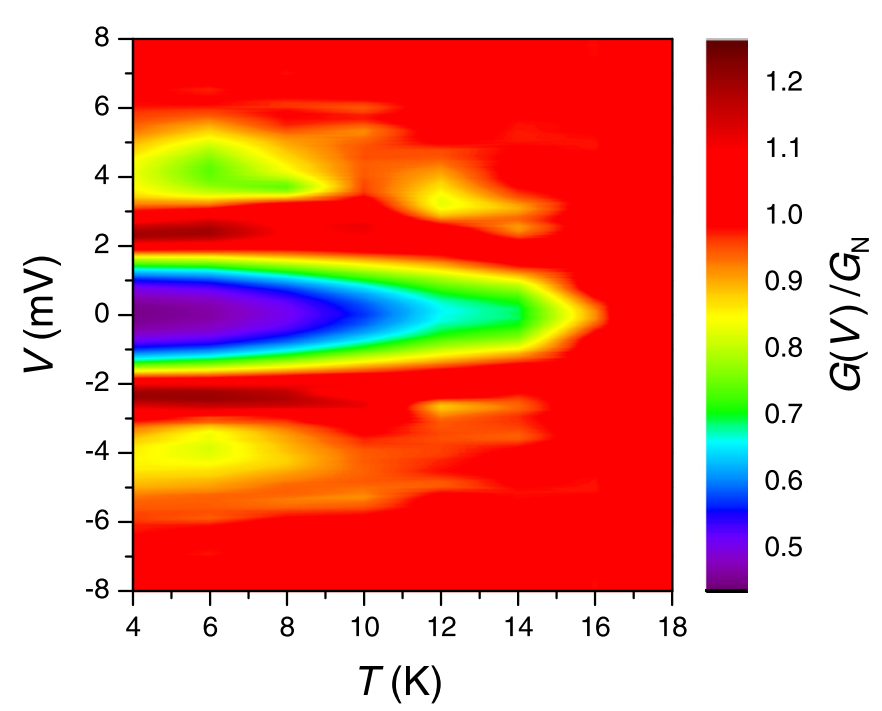

FIG. 6. Intensity plot of the differential conductance $G(V)$ as a function of temperature and applied bias voltage for the same device in Fig. 5. The peak and dip structures disappeared above $T_{\mathrm{c}}$, indicating that these structures originate from the superconductivity. 
reported values in PCAR spectroscopy by extrapolating down to $Z=0$. This is because (i) $P$ increases monotonically with the decrease in $Z$; (ii) ECAR spectroscopy can determine an ideal intrinsic $P$ value in ferromagnets, due to the ultra-clean superconductor/ferromagnet interface, when compared with PCAR spectroscopy. Here, we note that the finite $Z$ value in the ECAR devices might suggest the possibility of little interdiffusion at the $\mathrm{NbN} / \mathrm{Co}_{2} \mathrm{FeSi}$ interface by the annealing process to promote chemical ordering of the $\mathrm{Co}_{2} \mathrm{FeSi}$ layer. In addition, several reports exhibited that the half-metallicity can be realized for $\mathrm{Co}_{2} \mathrm{FeSi}$ by the Fermi level tuning on the substitution for other elements with different valence electrons. ${ }^{38-40}$ Indeed, a high MR ratio was reported at RT for the $\mathrm{Co}_{2} \mathrm{Fe}_{0.4} \mathrm{Mn}_{0.6} \mathrm{Si}$ based CPP-GMR devices. ${ }^{11,12}$ Hence, the quaternary Heusler alloys based on $\mathrm{Co}_{2} \mathrm{FeSi}$ are promising for half-metallic electrodes of superconducting spintronic devices.

In summary, we have demonstrated that Co-based Heusler alloy $\mathrm{Co}_{2} \mathrm{FeSi}$ was epitaxially integrated with superconductor $\mathrm{NbN}$ on the $\mathrm{MgO}$ substrate while keeping highquality magnetic and superconducting properties. The $\mathrm{NbN} /$ $\mathrm{Co}_{2} \mathrm{FeSi}$ layered ECAR devices including ultra-clean interfaces were fabricated by patterning from the epitaxial trilayer films. The average value of the spin polarization $\langle P\rangle$ for $\mathrm{Co}_{2} \mathrm{FeSi}$ was determined to be $54 \pm 2 \%$ with the finite $Z$ by ECAR spectroscopy, indicating that the intrinsic $P$ value in ECAR spectroscopy would exceed the reported values in PCAR spectroscopy. The combination of the highly spinpolarized Heusler alloy with the superconductor enables fundamental superconducting spintronic studies. Since the $P, p_{\mathrm{s}}$, and $T_{\mathrm{C}}$ of Co-based Heusler alloys can be controlled by the substitution of their constituent elements, this highly versatile superconducting spintronic system is also a candidate for practical superconducting spintronic devices.

See supplementary material for the additional figures of the conductance spectral shapes.

We would like to thank K. Makise, K.-i. Matsuda, S. Kawabata, Y. Asano, and Y. Tanaka for helpful advice and discussions. I.S. acknowledges support by JSPS KAKENHI Grant No. JP16K04933. A.A.G. acknowledges partial support by Ministry of Education and Science of the Russian Federation, Grant No. 14Y26.31.0007. This work was carried out under the Inter-University Cooperative Research Program of the Institute for Materials Research, Tohoku University (Proposal No. 16K0083). This work was performed at High Field Laboratory for Superconducting Materials, Institute for Materials Research, Tohoku University (Project No. 16H0028).

${ }^{1}$ A. I. Buzdin, Rev. Mod. Phys. 77, 935 (2005).

${ }^{2}$ J. Xia, V. Shelukhin, M. Karpovski, A. Kapitulnik, and A. Palevski, Phys. Rev. Lett. 102, 087004 (2009).

${ }^{3}$ T. Wakamura, H. Akaike, Y. Omori, Y. Niimi, S. Takahashi, A. Fujimaki, S. Maekawa, and Y. Otani, Nat. Mater. 14, 675 (2015).

${ }^{4}$ K. Senapati, M. G. Blamire, and Z. H. Barber, Nat. Mater. 10, 849 (2011).

${ }^{5}$ A. A. Golubov and M. Y. Kupriyanov, Nat. Mater. 16, 156 (2017).
${ }^{6}$ E. Goldobin, H. Sickinger, M. Weides, N. Ruppelt, H. Kohlstedt, R. Kleiner, and D. Koelle, Appl. Phys. Lett. 102, 242602 (2013).

${ }^{7}$ S. V. Bakurskiy, N. V. Klenov, I. I. Soloviev, M. Y. Kupriyanov, and A. A. Golubov, Appl. Phys. Lett. 108, 042602 (2016).

${ }^{8}$ K. E. Gray, Appl. Phys. Lett. 32, 392 (1978).

${ }^{9}$ J. Linder and J. W. A. Robinson, Nat. Phys. 11, 307 (2015).

${ }^{10}$ H.-x. Liu, T. Kawami, K. Moges, T. Uemura, M. Yamamoto, F. Shi, and P. M. Voyles, J. Phys. D: Appl. Phys. 48, 164001 (2015).

${ }^{11}$ J. Sato, M. Oogane, H. Naganuma, and Y. Ando, Appl. Phys. Express 4, 113005 (2011).

${ }^{12}$ Y. Sakuraba, M. Ueda, Y. Miura, K. Sato, S. Bosu, K. Saito, M. Shirai, T. J. Konno, and K. Takanashi, Appl. Phys. Lett. 101, 252408 (2012).

${ }^{13}$ A. Gupta, G. Singh, D. Kumar, H. Kishan, and R. C. Budhani, Appl. Phys. Lett. 103, 182602 (2013).

${ }^{14}$ D. Kumar, P. C. Joshi, Z. Hossain, and R. C. Budhani, Appl. Phys. Lett. 102, 112409 (2013).

${ }^{15}$ I. Shigeta, T. Kubota, K. Makise, S. Kimura, S. Awaji, B. Shinozaki, K. Koyama, K. Takanashi, and M. Hiroi, IEEE Magn. Lett. 8, 3305605 (2017).

${ }^{16}$ S. Imai, K.-i. Matsuda, T. Ishikawa, T. Uemura, and M. Yamamoto, Physica C 470, S851 (2010).

${ }^{17}$ R. J. Soulen Jr., J. M. Byers, M. S. Osofsky, B. Nadgorny, T. Ambrose, S. F. Cheng, P. R. Broussard, C. T. Tanaka, J. Nowak, J. S. Moodera, A. Barry, and J. M. D. Coey, Science 282, 85 (1998).

${ }^{18}$ G. J. Strijkers, Y. Ji, F. Y. Yang, C. L. Chien, and J. M. Byers, Phys. Rev. B 63, 104510 (2001).

${ }^{19}$ I. I. Mazin, A. A. Golubov, and B. Nadgorny, J. Appl. Phys. 89, 7576 (2001).

${ }^{20}$ B. S. D. Ch. S. Varaprasad, A. Rajanikanth, Y. K. Takahashi, and K. Hono, Appl. Phys. Express 3, 023002 (2010).

${ }^{21}$ B. Varaprasad, A. Rajanikanth, Y. Takahashi, and K. Hono, Acta Mater. 57, 2702 (2009).

${ }^{22}$ Y. Miura, K. Nagao, and M. Shirai, Phys. Rev. B 69, 144413 (2004).

${ }^{23}$ A. Shoji, S. Kiryu, and S. Kohjiro, Appl. Phys. Lett. 60, 1624 (1992).

${ }^{24}$ Z. Wang, A. Kawakami, Y. Uzawa, and B. Komiyama, J. Appl. Phys. 79, 7837 (1996).

${ }^{25}$ S. Wurmehl, G. H. Fecher, H. C. Kandpal, V. Ksenofontov, C. Felser, H.J. Lin, and J. Morais, Phys. Rev. B 72, 184434 (2005).

${ }^{26}$ I. Shigeta, O. Murayama, T. Hisamatsu, A. Brinkman, A. A. Golubov, Y. Tanaka, M. Ito, H. Hilgenkamp, and M. Hiroi, J. Phys. Chem. Solids 72, 604 (2011).

${ }^{27}$ I. Shigeta, Y. Sakuraba, S. Kimura, K. Koyama, K. Watanabe, K. Takanashi, and M. Hiroi, J. Magn. Soc. Jpn. 37, 222 (2013).

${ }^{28}$ S. Wurmehl, G. H. Fecher, H. C. Kandpal, V. Ksenofontov, C. Felser, and H.-J. Lin, Appl. Phys. Lett. 88, 032503 (2006).

${ }^{29}$ I. Galanakis, P. H. Dederichs, and N. Papanikolaou, Phys. Rev. B 66, 174429 (2002).

${ }^{30}$ B. Kang, H.-J. Kim, M.-S. Park, K.-H. Kim, and S.-I. Lee, Phys. Rev. B 69, 144514 (2004).

${ }^{31}$ A. A. Armenio, C. Cirillo, G. Iannone, S. L. Prischepa, and C. Attanasio, Phys. Rev. B 76, 024515 (2007).

${ }^{32}$ G. Sheet, S. Mukhopadhyay, and P. Raychaudhuri, Phys. Rev. B 69, 134507 (2004).

${ }^{33}$ Y. Ji, G. J. Strijkers, F. Y. Yang, C. L. Chien, J. M. Byers, A. Anguelouch, G. Xiao, and A. Gupta, Phys. Rev. Lett. 86, 5585 (2001).

${ }^{34}$ Z. Gercsi, A. Rajanikanth, Y. K. Takahashi, K. Hono, M. Kikuchi, N. Tezuka, and K. Inomata, Appl. Phys. Lett. 89, 082512 (2006).

${ }^{35}$ S. V. Karthik, A. Rajanikanth, T. M. Nakatani, Z. Gercsi, Y. K. Takahashi, T. Furubayashi, K. Inomata, and K. Hono, J. Appl. Phys. 102, 043903 (2007).

${ }^{36}$ S. Yamada, K. Hamaya, T. Murakami, B. Varaprasad, Y. K. Takahashi, A. Rajanikanth, K. Hono, and M. Miyao, J. Appl. Phys. 109, 07B113 (2011).

${ }^{37}$ L. Makinistian, M. M. Faiz, R. P. Panguluri, B. Balke, S. Wurmehl, C. Felser, E. A. Albanesi, A. G. Petukhov, and B. Nadgorny, Phys. Rev. B 87, 220402 (2013).

${ }^{38}$ B. Balke, G. H. Fecher, H. C. Kandpal, C. Felser, K. Kobayashi, E. Ikenaga, J.-J. Kim, and S. Ueda, Phys. Rev. B 74, 104405 (2006).

${ }^{39}$ T. Kubota, S. Tsunegi, M. Oogane, S. Mizukami, T. Miyazaki, H. Naganuma, and Y. Ando, Appl. Phys. Lett. 94, 122504 (2009).

${ }^{40}$ Y. Sakuraba, S. Kokado, Y. Hirayama, T. Furubayashi, H. Sukegawa, S. Li, Y. K. Takahashi, and K. Hono, Appl. Phys. Lett. 104, 172407 (2014). 